\title{
Could Government's R\&D Funding Improve Innovation Output of Public R\&D Institutes in China?
}

\author{
Jian-wen $\mathrm{HE}^{*}$ \\ Guangdong Institute of Scientific \& Technical Information, Guangzhou 510033, China \\ ${ }^{*}$ Corresponding author
}

Keywords: Government's R\&D funding, Public R\&D institutes, Innovation output, Co-Integration test, Granger cause.

\begin{abstract}
Based on co-integration model and Granger cause test, by using data from 2002 to 2016, this paper empirically analyzed the relationship between government's R\&D funding and innovation output of public R\&D institutes in China. The empirical results indicate that a long-term, stable and equilibrium relation exists between government's R\&D funding and public R\&D institutes' invention patent authorization, and furthermore the contribution from government's R\&D funding to public R\&D institutes' invention patent authorization is also significant in a short term; From the view of annual variation, the government's $R \& D$ funding is the Granger cause of public $R \& D$ institutes' granted invention patents, while the patents are not the Granger cause of government's R\&D funding; As a practical conclusion, the government's R\&D funding could expectedly improve innovation output of public R\&D institutes in China.
\end{abstract}

\section{政府研发资助能提高中国科研机构的创新产出吗?}

\author{
何健文 \\ 广东省科学技术情报研究所，广州，中国 \\ 通讯作者
}

关键词：政府研发资助；科研机构；创新产出；协整检验；格兰杰原因

摘要：以 2002-2016 年中国政府对国家科研机构的研发资助量和国家科研机构发明专利授权 量作为研究变量, 通过构造协整模型和运用格兰杰因果检验法, 分析了政府研发资助对国家 科研机构创新产出的影响。实证结论表明：（1）政府研发资助与国家科研机构发明专利授权 量之间具有长期稳定的均衡关系, 并且在短期内前者对后者能产生显著的贡献作用; (2) 政 府研发资助量变动是国家科研机构发明专利授权量变动的格兰杰原因, 但后者不是前者的格 兰杰原因;（3）在预期内, 中国政府研发资助能提高国家科研机构创新产出。

\section{1. 引言}

国家科研机构是指在国家财政预算资助体制和市场经济条件下，向全社会提供基础性、 战略性、公益性的科研成果产品或服务的科研组织 ${ }^{[1]}$, 按隶属关系分为中央部门属科研机构 和地方部门属科研机构两大类。国家科研机构是中国特色国家创新体系的重要组成部分, 也 是全社会各类创新主体之一。中国《“十三五” 国家科技创新规划》明确提出, 要发挥国家 科研机构在基础前沿和行业共性关键技术研发中的骨干和引领作用，提升国家科研机构的创 新主体能力。

创新投入和产出是衡量国家科研机构创新主体能力的两个重要方面。关于研发的统计表 明，中国政府投入到国家科研机构的研发资金在过去十多年里呈现出迅猛增长的态势，2016 年达到1 851.6 亿元, 相当于 2002年的6.7倍; 与此同时, 政府对国家科研机构研发资助额占 
国家科研机构研发经费总量的比重高达 $81.9 \%$ ，占政府对全社会的研发经费支出总额的 $60 \%$ ，相当于政府对企业研发资助的 4 倍。可见，政府资金是中国国家科研机构研发经费的 主要来源，中国国家科研机构同时也是政府研发资金的主要执行部门。中国政府对国家科研 机构的研发资助规模之大、增长之快，是否促进国家科研机构的创新产出、产生良好的创新 绩效? 从国内已有的研究和实践来看, 政府研发资助效果历来是政府和学界共同关注的重要 命题, 但关注的焦点往往局限于企业创新层面。鉴于此, 本文则对政府研发资助和国家科研 机构创新产出之间的作用机理进行分析, 以期填补相关研究的空白, 为政府更为科学、合理 地使用财政资金支持社会主体开展科研活动提供决策参考。

\section{2. 研究述评}

传统的研发投资理论主要讨论社会创新主体对自身研发活动的内部投入及相应的创新产 出效果。而现实中, 来自政府的资助对于社会创新主体研发活动的作用越来越重要, 使得学 者们对政府研发资助理论的关注度不断提高。

国外关于政府研发资助的理论集中围绕三个维度展开讨论：政府研发资助的方式; 政府 研发资助的影响因素; 政府研发资助的效果。一般而言, 政府研发资助主要有三种理论: (1) 市场失灵干预理论。该理论认为, 技术创新会形成知识溢出, 因此, 当研发活动的私人收益

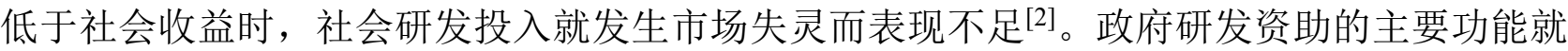
是通过降低技术创新成本从而有效缩小技术创新的私人边际收益率与社会边际收益率的差 距，以维持资助对象的研发动力 ${ }^{[3]}$ 。这一理论对于政府研发资助偏向于具有广泛技术溢出效 应的项目或组织而言具有积极意义。但是，该理论认为政府研发资助对私人投资存在替代效 应, 且没有充分考虑到专利保护对知识溢出的限制作用, 因而难以解释一些行业内部组织为 追求终身垄断专利而开展的研发投资行为。（2）融资约束松绑理论。该理论认为, 源于信息

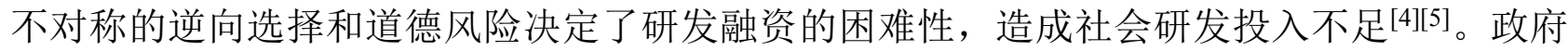
研发资助可以弥补这一短板，一方面直接缓解研发资金的社会需求，另一方面能分担研发活 动的高风险，从而降低研发项目或组织的融资约束。该理论指出了政府研发资助对市场投资 人的激励效应, 而且强调了社会创新主体的研发成果对于政府资助行为取向的重要性。 信号功能传递理论。该理论认为，政府研发资助发挥了鉴别研发项目或组织是否具备技术优 势和质量的重要作用，市场投资人根据政府行为决定是否对研发项目或组织给予资金支持 [6][7]。该理论将政府研发资助视作中介变量, 重点研究市场投资人、政府、研发主体的三方 博弯关系，但可能夸大了政府的甄别能力而忽视了如何激励政府选择出最优项目这一问题。

近年来，一些研究人员对中国的政府研发资助进行了探讨。舒红娟对中国的政府研发资 助规模和效果开展了评价研究, 指出国内的政府研发资助效果仍有改善的空间 ${ }^{[8]}$ 。周海涛、 张振刚通过实证研究发现, 作为国内政府研发资助的两种重要方式, 经费补助比税收优惠具 有更大的创新激励作用 ${ }^{[9]}$ 。朱斌认为，国内的政府研发资助具有一定的对象偏向性，要提高 政府研发资助的效率可从事前评估、事中监督和事后激励着手 ${ }^{[10]}$ 。白俊红、市元超指出, 区 域基础设施环境、人力资本环境、金融环境以及制度环境对政府研发资助均具有显著的正面 促进作用，但经济发展环境的影响效应并不显著 ${ }^{[11]}$ 。

总体而言，上述研究主要考察政府研发资助与企业创新的关系，而鲜有探讨政府研发资 助对科研机构、高校等其它创新主体的影响作用。而相关实证研究的结论则表明, 政府研发

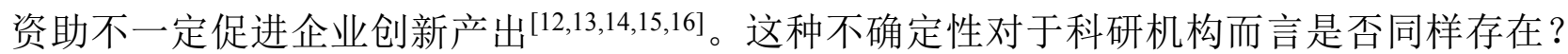
中国政府研发资助能否提高国家科研机构的创新产出？基于此，本文以国家科研机构作为研 究对象，围绕中国政府研发资助的效果问题，通过协整分析、建立误差修正模型以及格兰杰 因果关系检验，实证考察政府研发资助和国家科研机构创新产出之间的长期及短期关系。 


\section{3. 研究设计}

本文的研究变量有两项：政府研发资助和国家科研机构创新产出。关于前者，本文参考 Brown $^{[5]}$ 、Kleer ${ }^{[6]}$ 、梁莱歆 ${ }^{[9]}$ 等人的做法, 采用国家科研机构研发经费内部支出中来源自政 府资金的部分作为相应的测度指标, 并对其流量数据（GInsRD）取对数（LNGInsRD）; 关 于后者, 本文借鉴王俊 ${ }^{[16]}$ 、Mario ${ }^{[17]}$ 等人的做法, 采用国家科研机构的发明专利授权量作为 相应的测度指标, 并对其流量数据（InsIPTN）取对数（LNInsIPTN）。上述指标及基础数据 均选自《中国科技统计年鉴》《中国统计年鉴》，数据的时间跨度为2002-2016年。取对数后， 这两项变量随时间变化而呈现的趋势如图1所示。

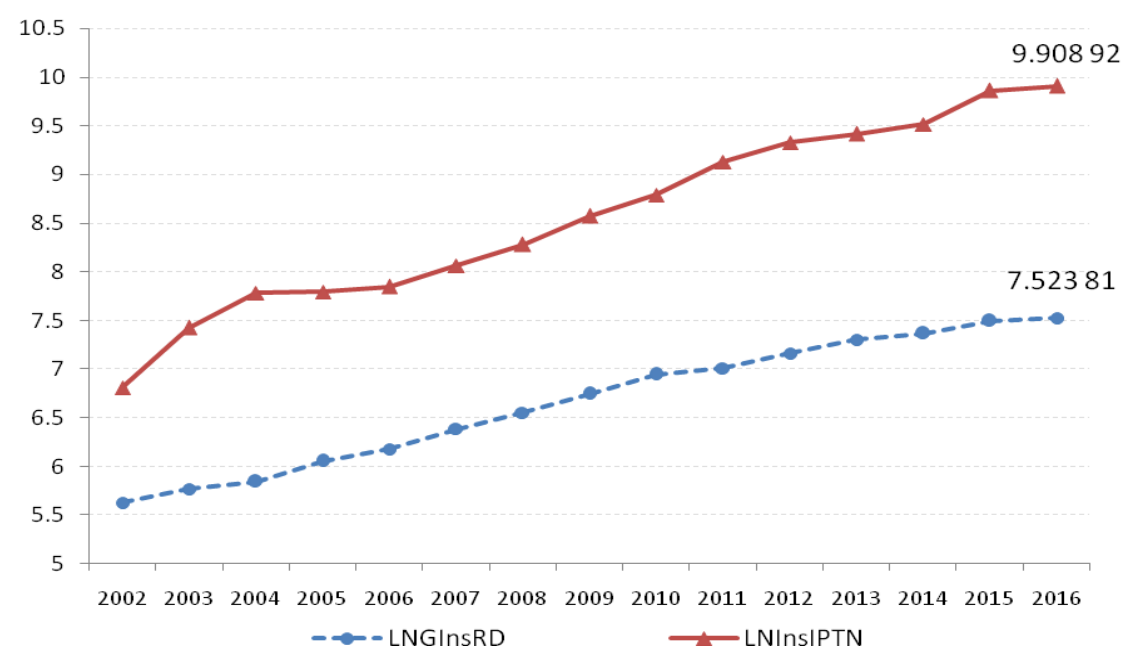

图1２002-2016年LNGInsRD与LNInsIPTN的变化走势

\section{4. 研究过程}

\section{1 单位根检验}

首先, 要检验变量的平稳性, 这是进行协整分析的前提。一般以是否存在单位根作为判 别平稳性的依据。本文要进行单位根检验的是时间序列变量LNGInsRD与LNInsIPTN以及它们 的一阶差分变量DLNGInsRD与 DLNInsIPTN。通过使用ADF检验法 ${ }^{[18]}$, 可以得到如下表所示 的检验结果。

表1 变量平稳性的检验结果

\begin{tabular}{ccccc}
\hline 变量 & $\mathrm{ADF}$ 值 & 检验类型 $(\mathrm{c}, \mathrm{t}, \mathrm{k})$ & 临界值 & 结论 \\
\hline LNGInsRD & -1.227660 & $(\mathrm{c}, \mathrm{t}, 0)$ & $-3.362984^{*}$ & 非平稳 \\
LNInsIPTN & -4.642562 & $(\mathrm{c}, \mathrm{t}, 1)$ & $-3.875302^{* *}$ & 平稳 \\
DLNGInsRD & -3.814765 & $(\mathrm{c}, 0,0)$ & $-3.144920^{* *}$ & 平稳 \\
DLNInsIPTN & -4.613075 & $(\mathrm{c}, 0,1)$ & $-3.175352^{* *}$ & 平稳 \\
\hline
\end{tabular}

注：ADF检验类型（c，t， k）中， c表示常数项，t表示时间趋势项， $\mathrm{k}$ 表示滞后期; *表示该临界值 的显著性水平为 $10 \%, * *$ 表示该临界值的显著性水平为 $5 \%$ 。

可见, $L N G I n s R D$ 为非平稳序列变量, LNInsIPTN为平稳序列变量, 而 $D L N G I n s R D$ 与 $D L N I n s I P T N$ 均为平稳的。因此, LNGInsRD与LNInsIPTN均为一阶单整序列, 两者之间可能 存在协整关系, 即它们可能会构成平稳的线性组合。这表明, 两个序列之间可能保持着长 期、稳定的关系。 


\section{2 协整检验}

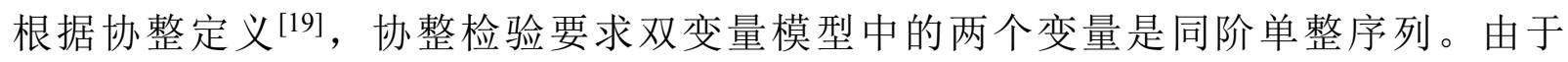
$L N G I n s R D$ 与LNInsIPTN均为一阶单整序列 I (1), 故满足上述要求。采用 E-G两步法, 对 2002-2016年中国政府对国家科研机构的研发资助量和国家科研机构发明专利授权量之间是 否存在协整关系进行验证。

(1) 方程拟合。采用OLS方法估测协整向量。运用计量学分析工具EViews 6.0, 可以得 到以下方程:

$$
\text { LNInsIPTN }=-0.7490+1.3968^{*} L N G I n s R D+\varepsilon t
$$

其中, $\mathrm{R}^{2}=0.9671$, 校正后的 $\mathrm{R}^{2}=0.9644, \mathrm{~F}=352.6871$ 。则协整向量可能是 $(1,-1.396$ $8,0.7490)$ 。

(2) 残差序列的平稳性检验。由上述方程可得, 待估计的残差变量为:

$$
\varepsilon t=L N I n s I P T N-1.3968 * \text { LNGInsRD }+0.7490
$$

经检验, $\mathrm{ADF}$ 检验值低于显著性水平 $1 \%$ 下的临界值（见表 2 ）, 故在该水平下残差序列 $\varepsilon \mathrm{t}$ 没有单位根。由此可知, 残差是平稳变量。又因为 $L N G I n s R D$ 与 $L N I n s I P T N$ 均为一阶单整序 列，所以二者具有协整关系，协整向量是（1，-1.396 8，0.749 0)。

表 2 残差平稳性的检验结果

\begin{tabular}{ccccc}
\hline 变量 & $\mathrm{ADF}$ 值 & 检验类型 $(\mathrm{c}, \mathrm{t}, \mathrm{k})$ & 临界值 & 结论 \\
\hline$\varepsilon_{t}$ & -3.173555 & $(0,0,0)$ & $-2.754993 * * *$ & 平稳 \\
\hline
\end{tabular}

注：本表中 $\mathrm{c} 、 \mathrm{t} 、 \mathrm{k}$ 的含义参照表 $1 ; * * *$ 表示该临界值的显著性水平为 $1 \%$ 。

协整检验的结果表明: 中国政府对国家科研机构的研发资助和国家科研机构的发明专利 授权量之间存在长期、稳定的关系; 从较长周期看, 政府研发资助对国家科研机构发明专利 授权量的弹性为 1.3968 , 即政府研发资助流量每增长 $1 \%$, 相应地, 国家科研机构的发明专 利授权量增长约 $1.40 \%$ 。这反映出政府研发资助对国家科研机构创新产出具有较为明显的提 升效用。

\section{3 误差修正模型构造}

由于残差项 $\varepsilon t$ 已通过单位根检验, 可以明确政府研发资助与国家科研机构发明专利授权 量之间存在长期、稳定的协整关系，故下一步将对两者的关系构造误差修正模型（ECM）, 以建立对长期偏离的一种纠正机制。模型的初始形式如下:

$$
\Delta L_{N I n S I P T N}=C+\beta^{*} \Delta L N G I n s R D_{t}+\gamma^{*} E C M_{t-1}+\varepsilon t
$$

其中,

$$
\operatorname{ECM}_{t-1}=\operatorname{LNInsIPTN}(-1)-1.3968 * \operatorname{LNGInsRD}(-1)+0.7490
$$

按照传统的建模方法 ${ }^{[20]}$, 本文先选定3阶的滞后变量, 然后再对一些不显著的变量进行 逐个排除, 最终获得估计后的误差修正模型如下所示:

$$
\begin{aligned}
\text { DLNInsIPTN }= & -0.7496 * D L N I n s I P T N(-1)-1.1360 * D L N G I n s R D(-1)+1.2073 * D L N G I \\
& n s R D(-2)+1.9153 * D L N G I n s R D(-3)-0.9130^{*} E C M_{t-1}
\end{aligned}
$$

其中, $\mathrm{R}^{2}=0.9207$, 校正后的 $\mathrm{R}^{2}=0.8572$, D.W. $=3.1315$

通过上述分析, 可以得到三方面的推论: 系。

(1) 从长期来看, 政府研发资助和国家科研机构发明专利授权量之间存在着动态均衡关 
(2) 从短期来看，国家科研机构发明专利授权量的变动受到自身和政府研发资助的变动 的共同影响。其中，在 $5 \%$ 的显著性水平下，滞后 $1 、 2 、 3$ 年的政府研发资助的变动、滞后 1 年的国家科研机构发明专利授权量的变动对国家科研机构发明专利授权量的变动产生显著的 影响。

(3) 在本文的误差修正模型中，误差修正项 $E C M_{t-1}$ 的系数在 $5 \%$ 显著性水平下为 -0.913 0 , 说明政府研发资助和国家科研机构发明专利授权量之间的上期非均衡误差对国家科研机 构发明专利授权量当期波动的影响是显著的, 意味着政府研发资助和国家科研机构发明专利 授权量的短期波动在一定程度上偏离了二者的长期均衡关系; 该系数的绝对值接近 1 , 说明 上期非均衡误差对国家科研机构发明专利授权量当期波动的调整幅度较大, 意味着本模型自 身将偏离均衡误差调整、修正到均衡状态的能力较强。

\section{4 格兰杰因果关系检验}

根据协整分析的结果，长期、稳定的均衡关系在中国政府研发资助与国家科研机构创新 产出之间是存在着的, 但其并不等价于因果关系。为此, 本文采用格兰杰因果关系检验法做 进一步验证。鉴于格兰杰因果检验法要求待检验的变量必须是平稳的, 故本文选用变量 DLNGInsRD 和DLNInsIPTN所对应的年度数据进行检验, 得到如下表所示的结果。

表3 政府研发资助与国家科研机构发明专利授权量的格兰杰因果检验

\begin{tabular}{|c|c|c|c|c|}
\hline 滞后期（年） & & 1 & 2 & 3 \\
\hline 假 设 1 : DLNGInsRD 不 是 & $\mathrm{F}$ 统计量 & 0.08279 & 0.60575 & 14.37830 \\
\hline DLNInsIPTN 的格兰杰原因 & 显著性水平 & 0.78010 & 0.57590 & 0.02760 \\
\hline 假 设 2 : DLNInsIPTN 不是 & $\mathrm{F}$ 统计量 & 0.10142 & 0.41298 & 0.28914 \\
\hline$D L N G I n s R D$ 的格兰杰原因 & 显著性水平 & 0.75740 & 0.67910 & 0.83230 \\
\hline
\end{tabular}

可见, 当滞后期为 3 年时, 假设 1 的显著性水平低于 0.05 , 故拒绝原假设, 即: 政府研发 资助量的变动是国家科研机构发明专利授权量变动的格兰杰原因。而在滞后期分别为 $1 、 2 、 3$ 年时，国家科研机构发明专利授权量变动均不是政府研发资助量变动的格兰杰原因。

\section{5. 研究结论及政策建议}

\section{1 基本结论}

本文采用2002-2016年中国政府对国家科研机构的研发资助量和国家科研机构发明专利 授权量数据, 通过协整检验、误差修正模型构造和格兰杰因果检验, 对政府研发资助和国家 科研机构创新产出之间的长期关系及短期动态因果关系进行实证分析, 得到了以下几点基本 结论并做出了相应解释:

中国政府研发资助和国家科研机构发明专利授权量之间构成了长期、稳定的均衡关系。 而在本文所选定时限的短期内, 滞后3年的政府研发资助量变动是国家科研机构发明专利授 权量变动的格兰杰原因, 但滞后3年的国家科研机构发明专利授权量变动不是政府研发资助 量变动的格兰杰原因。

（1）政府研发资金流量变动是国家科研机构发明专利授权量变动的格兰杰原因。与 $\mathrm{Mario}^{[17]}$ 、 $\mathrm{Paul}^{[21]}$ 等人研究得出的结论一致, 本文的研究结果显示, 政府研发资金能够提升 国家科研机构的创新产出, 从而证实了对于公共科研机构而言政府研发资助具有激励效应和 产出效率。政府研发资助对国家科研机构创新产出的促进作用主要依靠两种路径来实现 ${ }^{[22,23]}$ : 一是对科研人员物质需求的满足, 使科研人员的工作积极性和满意度大幅提高, 从而 形成对其研发活动效率的正向激励作用; 二是对技术设备的更新、改造和升级, 使科研机构 
具备技术资源的硬件优势，有利于开展前沿或新兴技术的学习和攻关。当一系列具有新颖 性、创造性、实用性的科研成果得到开发、转让、扩散以至上升为行业标准时, 科研机构为 保障自身的合法收益, 会对这些科研成果申请知识产权保护, 从而使科研机构形成并拥有以 发明专利产出的数量规模为表征的创新产出。

(2) 国家科研机构发明专利授权量变动不是政府研发资助流量变动的格兰杰原因。国外 的政府治理理论表明, 因素配置法是政府调控社会公共资源的基本手段, 而社会创新主体获 得的科研成果是甄别其创新产出水平的重要标志, 是政府采用因素配置法来施行创新资助政 策的主要依据 ${ }^{[24,25]}$ 。因此, 科研机构必须积极开展科研活动并实现成果产出, 在争取公共创 新资源的过程中形成独特的竞争优势, 才能有机会获得政府研发资助。本文认为, 中国政府 对科研机构的研发资助, 主要目的是为了维持国家科研系统的稳定、夯实其研发力量、鼓励 其持续开展研发活动, 从而促使其提升创新产出以应对全球产业变革带来的挑战和满足未来 社会发展对科学技术成果储备的迫切需求, 而不是在科研机构具备了创新产出优势之后才给 予重点扶持。

\section{2 政策建议}

国家科研机构的创新产出本质上是科研人员的创新产出, 而科研人员的创新产出具体表 现为他们所创造的科研成果。由于科研成果的产出往往以一定的创新投入为前提, 投入的强 度和稳定性与产出的规模、质量和效率都有着密切的关系, 对于科研机构创新产出的优化调 整起着重要作用。已有研究表明, 由于研发活动存在“市场失灵”和“溢出”效应, 国家科研机 构（尤其是基础研究和应用研究机构、共性技术研究机构）从事研发活动难以从市场获得足 够的经济收益。对中国而言, 政府采用稳定性支持的方式, 对这些科研机构开展研发资助, 恰恰弥补了上述短板, 能够确保科研机构研发投入的持续性, 有利于科研人员专业素质的培 养, 从而提升国家科研机构的创新动能。

与此同时，国家科研机构应当促进科研成果转化扩散进程，提升科研成果的经济社会应 用价值。自2002年以来，中国国家科研机构的发明专利授权量的年均增长率为 $26.5 \%, 2016$ 年达到 2.01 万件。尽管发明专利授权量实现了大幅增长, 但由于在过去一段较长的时间里, 国内科研管理存在“重科研过程、轻经济社会绩效”等种种问题, 相当一部分专利仅停留在实 验室阶段, 基本没有实现直接或间接的转移转化, 难以成为有效传播的行业知识或技术标 准, 从而无法创造出应有的经济社会效益。因此, 将科研成果转化率纳入到国家科研机构的 成果绩效评价考核体系中, 并逐步推广建立政府与科研机构之间的成果约定购买制度, 使得 政府研发资助的投入机制从现行关注立项申请的“期货式”转变为关注成果的真实性、新颖性 和经济社会价值的“现货式”，将有利于建立健全国家科研机构研发创新的政府引导机制，促 进社会产业分工迈向价值链中高端, 从而也为政府合理调配研发资金提供重要方向和有力依 据。

《国家创新驱动发展战略纲要》提出, 要推动资源配置从以研发环节为主向产业链、创 新链、资金链统筹配置转变。因此, 面向国家科研机构, 通过逐步建立基于科研成果及其转 化应用效果的政府研发资助制度, 为政府加大研发资助力度创造有利条件, 通过完善落实国 家重点研发计划项目的经费使用和分配政策, 以充分调动国家科研机构人员的创新能动性, 是符合国家的宏观战略部署的。在目前的创新环境下, 中国政府对科研机构的研发资助不仅 有助于形成科研机构基础研究活动的稳定性, 提升科研人员的专业技术水平, 还可以为集中 力量开展基础前沿和行业共性关键技术研发攻关，建设世界一流科研机构奠定基础。政府对 企业的研发资助可以在一定程度上促进企业的创新投入，但是否促进企业创新产出的提高目 前还没有统一结论; 并且, 企业获得政府研发资助的最终目的是获得经济利润, 而不是自有 技术的推广应用。中国政府不能对来自企业的前沿基础或行业共性技术的研发攻关抱有太高 的期待。 


\section{致谢}

本文为广东省省级科技计划项目 《云计算与大数据产业技术信息智能跟踪和决策支撑系 统开发及应用研究》（编号：2017A010106008）的阶段性成果之一。

\section{References}

[1] CHENG Ru-yan. Analysis on main countries' funding systems of public research institutes[J]. Global Science, Technology and Economy Outlook, 2016(10):1-5.

[2] ROMER P M. Endogenous technological change[J]. Journal of Political Economy, 1990(98):71-102.

[3] GUELLEC, POTTELSBERGHE. The impact of public R\&D expenditure on business R\&D[J]. Economic Innovation New Technology, 2003, 12(3):225-243.

[4] STIGLITZ, JOSEPH E, ANDREW W. Credit rationing in markets with imperfect information[J].American Economic Review, 1981, 71:393-410.

[5] BROWN, MARTINSSON, PETERSEN. Do financing constraints matter for R\&D?[J]. European Economic Review, 2012(56):1512-1529.

[6] KLEER. Government R\&D subsidies as a signal for private investors[J]. Research Policy, 2010, 39:1361-1374.

[7] TAKALO T, TANAYAMA T. Adverse selection and financing of innovation: is there a need for R\&D subsidies?[J]. Journal of Technology Transfer, 2010, 35(1): 16-41.

[8] Shu Hong-juan. Performance evaluation and improvement research on public R\&D institute' funding in China[D]. Nanjing University of Aeronautics and Astronautics, 2011: 94-106.

[9] ZHOU Hai-tao, ZHANG Zhen-gang. The effects of different ways of government R\&D investment on enterprise innovation investment and performance[J]. Chinese Journal of Management, 2015, 12(12):1797-1804.

[10]ZHU Bin. Government R\&D subsidies should be provided with universality[N]. Dazhong Daily, 2015-05-06(10).

[11]BAI Jun-hong, BIAN Yuan-chao. Research on the spatial- autocorrelation of China's government R\&D subsidies[J]. Science Research Management, 2016, 37(1):77-83.

[12]PAUL A D, BRONWYN H H, ANDREW A T. Is public R\&D a complement or substitute for private R\&D? A review of the econometric evidence[J]. Research Policy, 2000, 29:497-529.

[13]LIANG Lai-xin, MA Ru-fei, TIAN Yuan-fei. R\&D financing source and technical innovation-Empirical research on China's large and medium-size industry firms[J]. Science of Science and Management of S.\& T. , 2009(7): 89-93.

[14]LI Zhang, SU Jun. An empirical study on the signal effect of R\&D subsidy: Evidence from 863 program[J]. China Soft Science Magazine, 2017(2): 54-65.

[15]CHENG Hua, ZHAO Xiang. Effect of government science and technology subsidies on enterprise $R \& D$ output - an empirical analysis based on the large and medium-sized industry enterprise of our country[J]. Studies in Science of Science, 2008, 26(3): 519-526.

[16] WANG Jun. The experiential study on the impact of government R\&D subsidies on the business R\&D input and innovation output[J]. Studies in Science of Science, 2010, 28(9): 1368-1374. 
[17]MARIO C. New models for measuring the R\&D performance and identifying the productivity of public research institutes[J]. R\&D Management, 2004, 34(3): 267-280.

[18]DAVID A D, WAYNE A F. Distribution of the estimators for autoregressive time series with a unit root[J]. Journal of the American Statistical Association, 1979, 74(366): 427-431.

[19]ENGLE R, GRANGER C. Co-integration and error correction: repressentation, estimation and testing[J]. Econometrics, 1987(55): 251-276.

[20]GAO Tie-mei. Econometric analysis method and modeling: application of EViews and examples[M]. Beijing: Tsinghua University Press, 2016: 56-98.

[21]PAUL A D, BRONWYN H H. Heart of darkness: modeling public-private funding interactions inside the R\&D black box[J]. Research Policy, 2000(29): 1165-1183.

[22]FAN Li-hong. Experience and enlightenment of foreign funding for public scientific research institutions[J]. Forum on Science and Technology in China, 2007(5): 33-37.

[23]GOOLSBEE A. Does government R\&D policy mainly benefit scientists and engineers?[J]. American Economic Review, 1998, 88(2): 298-302.

[24]CHEN Dong-hua, ZHANG Tie-sheng, LI Xiang. Law environment, government regulation and implicit contract: Empirical evidence from the scandals of Chinese listed companies[J]. Economic Research Journal, 2008(3): 60-72.

[25]GINO F, PISANO G. R\&D Portfolio Strategy and Performance: A Behavioral Model[J]. Blazing Saddles, 2008(1): 43-47. 